\title{
ICT-based sub-practices in sustainable development of city transport
}

\author{
Greger Henriksson \\ Division of Environmental Strategies Research and \\ Centre for Sustainable Communications \\ KTH Royal Institute of Technology \\ Stockholm, Sweden \\ gregerh@kth.se \\ Mattias Höjer \\ Division of Environmental Strategies Research and \\ Centre for Sustainable Communications \\ KTH Royal Institute of Technology \\ Stockholm, Sweden \\ hojer@kth.se
}

\begin{abstract}
How could use of ICTs make transport systems in large cities sustainable? To what degree would then everyday patterns of action, in which use of ICT is becoming increasingly integrated, be an effective force in such sustainable development? In this paper we use own and other ethnographic studies of how people travel and plan their travel in large Nordic cities and explore a couple of scientific articles built around scenarios of sustainable development of ICT-supported transport in large cities. In the scenarios the role of ICT was most prominently for travel planning, collective ownership and/or access to vehicles, payment systems and traffic management for environmental purposes. Two of the scenario articles were selected for a closer analysis. In the ethnographic material sub-practices, critical for transport in everyday life, were identified. The aim was to show if and how proposed solutions in the sustainability scenarios are possible and meaningful for urban dwellers to integrate into their everyday travel practice.

We found that scenarios of this kind might benefit from being supplemented with certain components. We discuss how actors that currently develop traffic management, mobility and travel planning services are somewhat blind to some significant parts of the whole spectrum of relevant social practices. We also discuss how this could provide conditions for actors and stakeholders to highlight and facilitate sub-practices with ICT use and in line with sustainable development. We tentatively discuss and propose that authorities responsible for urban transport set goals for maintaining and spreading certain practices, related to sustainability impacts. In this way the development of everyday social practice among city residents could be supported as an effective force in the development of the city's transport system, specifically including the development of ICT in relation to transportation.
\end{abstract}

Index Terms - Social practices, everyday life, ethnography, ethnology, environmental sociology, urban transport, travel planning, travel information services, ICT, scenarios, sustainability, user-centred perspective

\author{
Anders Gullberg \\ Division of History of Science, Technology and Environment \\ and Centre for Sustainable Communications \\ KTH Royal Institute of Technology \\ Stockholm, Sweden \\ anders.gullberg@urbancity.se \\ Åsa Nyblom \\ Division of Environmental Strategies Research and \\ Centre for Sustainable Communications \\ KTH Royal Institute of Technology \\ Stockholm, Sweden \\ asa.nyblom@abe.kth.se
}

\section{Introduction}

There are problems in city traffic in terms of environmental impacts and in terms of that people who travel in big cities experience problem to arrange their accessibility and travel satisfactorily, see e.g. [1]. At the same time use of ICT is getting an increasing role for travel in the city and the planning of this. It has been suggested that ICT could be used to solve both environmental problems and city travelers' problems of arranging their accessibility and travel satisfactorily. An expanding field of research concerns systems for multimodal travel planning currently in operation. Some systems have been investigated in recent studies, regarding potentials to support sustainability-oriented decisions [2].

The aim of this paper is to illustrate the variety and wide range of practices involved in shaping everyday travel in cities. The aim is further to use this illustration to explore some existing scenarios of urban travel with a sustainability perspective and a high degree of depicted ICT use. On the basis of our theoretical perspective (see below) we postulate that a comprehensive transformation of urban travel would include the full range of related practices. Measures for sustainable development of city transport risk failing if some relevant practices are not targeted. Our aims are approached by trying to respond to the following questions:

- How can the range of social practices related to urban travel be pictured, by identifying a handful of specific practices at different 'end-points' of that range, to provide a better basis for developing scenarios and measures for sustainable urban travel with ICT?

- How are contemporary scenarios addressing ICT-use in relation to how social practices shape urban transport? 


\section{A. Methodology}

Development of everyday social practices in cities, including possible use of ICTs, shape urban traffic [3]. In line with Social Practice Theory (SPT) the entire system of what people do in the city can be said to consist of sub-processes, i.e. of different social practices. Social practices should be seen as co-evolving with sociotechnical systems, economic conditions etc. [4]. Furthermore, travel is dependent on how a wide range of practices evolves, e $\mathrm{g}$ in social, family or work life [5]. How practices evolve can therefore affect cities, with similar economic development and geography, getting e g different relative proportions of car traffic, public transport cycling and walking.

In relation to travel, the so called activity approach [6] is a more established methodology than social practice theory. The activity approach views travel as depending on various, socially constructed, needs and demands in everyday life [7]. Activities and travel are justified by needs, commitments, demands etc., but travel is also dependent on the potential and opportunities people actually have to fulfil them. People connect to other and get access to different resources and activities through transportation and communication. Transportation and communication is thus interwoven with everyday life and commitments $[8,9,10]$.

The activity approach can be used in combination with SPT $[6,11]$. Both methodologies take a "situational approach" [6] to travel patterns, viewing individual trips as parts of "highly complex series of interrelationships of various trips, in-home, out-of-home and household activities" [6]. Therefore we lean also on the activity approach in this paper, when focusing on travel in terms of social practices. By extending the activity approach with SPT, we emphasize the importance of looking at the context in which the activities are played out, which we argue gives a broad understanding of what travel practice is.

In line with SPT we will refer to bundles or complexes of practices [12], where practices linked in bundles or complexes enable people to handle e $\mathrm{g}$ all the activities and travel of a day in life. In addition to this [12] we will use the term subpractices, and regard these as being bundled etc. with other sub-practices, as well as with more comprehensive practices, e $\mathrm{g}$ the practice of using public transport or car on a daily basis. With sub-practices, we mean limited but meaningful parts of activity patterns through which the urban citizens handle recurring situations in their daily lives.

We build this paper on mainly two types of material. First, we look into a handful of articles concerning smart cities and/or scenarios concerning urban traffic $[13,14,15,16]$. A requirement when choosing articles to explore was that they had both environmental sustainability and ICT as decisive and characteristic parts. The ICT-parts were focused on areas like planning travel, collective ownership, payment systems and traffic management for environmental purposes. After a brief exploration we selected two articles most fit for our purpose $[15,16]$.

Second, we used own, and others', previous ethnographic studies of how people travel and plan their trips in relation to their planned activities $[5,13,14,15,16]$. These were made in
Nordic larger city contexts, and in this paper we have a special focus on Stockholm as example. In the ethnographic studies, we first identified sub-practices related to critical points and difficulties in everyday travel practice.

\section{B. ICTs and sustainable development of city transport}

Our view on sustainable development includes consideration of the risk that new large-scale technologies will counteract sustainable development, by giving rise to new, large-scale environmental impacts [12]. Therefore, technical improvements of environmentally harmful activities need to be accompanied by some kind of limiting conditions or measures. ICT has a potential for both those cases - it already works as a lubricant to different activities but also as a limiting technology. ICT is put to a limiting use by enterprises, organizations and authorities, e.g. since the user has to register, $\log$ in, pay etc., in order to access information, means of communication, services and products. Thus limitations of environmental impacts of travel could come from legislation mediated by ICT:s, e.g. congestion charging or parking facilities. Limitations also come from the city's infrastructure and physical form as well as from natural or market restrictions in access to energy and other natural resources. A point of departure for this paper is that when such limitations of environmental impacts are operating, new uses of ICT could contribute to ways of planning, using and providing city transport in line with sustainable development.

Taking into account the high impact development of ICT currently has on everyday life, it becomes highly interesting to connect how practices shaping city traffic could evolve in relation to increased use of ICTs in urban daily life, especially for transport related practice.

\section{Scenarios}

Two scenarios of sustainable development of city transport with ICT were selected for our analysis. The first was the "post-car system", developed by sociologist and mobility researcher John Urry [15]. In this explorative scenario, focused on transformation rather than goal-fulfilment, Urry identified 'six technical-economic, policy and social transformations that in their dynamic interdependence might tip mobility into a new system, the post-car' [15: p 33]. Of most interest here are the last four of the transformations which Urry presented: the development of smart-card technology; cars generally being de-privatized; transport policy shifting away from predict-andprovide models towards changing driver behavior through demand-reduction strategies; and communications and the Internet galaxy becoming increasingly interconnected with transportation [15: 34-35, italics in original]. After presenting the six transformations Urry concludes:

So there are six sets of changes that I have briefly outlined. None of them is sufficient in themselves to tip the car system into new channels. But my proposal is that their interdependencies occurring in an optimal order might thus provoke the emergence of a post-car system. A series of 
small changes now might produce a sense of contagion as many changes sweep through the system. [15: 35]

Urry's line of argument is based also on other theorists in the field, and starts from the term "automobility". He refers to others who e.g. argue that: "a car is not a car because of its physicality but because systems of provision and categories of things are materialized in a stable form', and this generates the distinct affordances that the car provides for the hybrid of the car driver" [15: 26]. Urry discusses how the system of automobility could be seen as spreading as an in some sense viral but basically complex and non-linear system [15, p. 27]. The line of argument in Urry's introduction ends in his analysis that 'from relatively small causes an irreversible pattern was laid down and this ensured the preconditions for automobility's self-expansion over the past astonishing century' and that this is based in e.g. "automobility's exceptional power to remake time-space, especially because of its peculiar combination of flexibility and coercion" [15].

In his conclusion Urry takes up this line of argument again and argues that through "complexity approaches" he emphasizes three points about "a shift away from the current car system". These points are: 1) 'any post car-system will substantially involve the individualized movement that automobility presupposes and has simultaneously brought into being as an irreversible consequence of the century of the car' [15: 36].; 2) The system of automobility will suddenly disappear and "become like a dinosaur, housed in museums"; 3) "this tipping point / ---/ cannot be read off from linear changes in existing firms, industries, practices and economies. Just as the Internet and the mobile phone came from nowhere, so the tipping point towards the post-car will emerge unpredictably. It will probably arrive from a set of technologies or firms or governments that are currently not a centre of the car industry and culture" [15: 36].

The other selected scenario $I^{2} P S U T-$ an Integrated Information and Payment System in Urban Transport [16], put forward by Anders Gullberg, is goal-oriented and normative, focusing on a future state rather than a transformation process itself. I ${ }^{2}$ PSUT is argued to have 'the potential to reduce information deficits, improve coordination, and harness unused capacity' for the benefit of urban residents as well as in order to reach environmental goals. According to Gullberg's scenario, those who want, ask a functionally extended journey planner for alternatives (e.g. modes, travel time, price, uncertainty, carpooling, car sharing, car hire, taxis, forwarding agent, comfort, reservation of rental bike or parking space, alternative and equivalent destinations and equivalent service without physical transfer). Prices vary with time, space and travel mode in such a way that congestions almost disappear, travel times become predictable (and usually shorter), and environmental policy can be supported. When the user's choice is made it means that provider(s) and the customer have entered into a contract with each other [16].

Furthermore, Gullberg includes navigation assistance during the whole trip for all modes of transport including walking and cycling and for freight transportation as well as for public transport and car traffic. The providers - public transport bodies and/or road managers - mobilize additional resources to fulfil its obligations. When the destination of a trip is reached, evaluation is used by both the consumer and the supplier to improve subsequent decisions and forecasts. In case of significant deviation automatic compensation will be given, which creates an incentive for suppliers to improve forecasts and offerings. Road users, especially drivers will be invited as co-producers and are offered financial rewards for passengers and fright. The system is open including for joint transport and for different groups of like-minded people (social communities) and for the development of additional services, e.g. priority seating on public transport or priority lanes on the highway, and infinitely more possibilities.

Urban transport fees can be levied for negative externalities of congestion, parking lots, local and global environmental impact, risk of accidents, noise, traffic injustice and urban sprawl. Positive effects can occur even with limited participation in the $I^{2} P S U T$ system thanks to the highly valuable information generated by users' queries, concluded agreements and trips made. Increased travel due to improved terms can be balanced by means of coordinated transport prices for an entire urban region while continuing sprawl also can be countered by pricing.

Gullberg envisions that in his system requirements will escalate to producers through a strong consumer, client and innovation pressure, while making large savings by greatly reduced needs for investment and efficient use of the vehicle fleet and infrastructure. Consumers are offered improved and new services with substantially higher quality, new systems can be developed and chaos be avoided by coordinating the customers and affect the influx to the various transportation services. Ultimately, he sees how the long-term development of cities is affected.

In the short term, the solutions proposed in the scenario would achieve shorter and predictable travel and transport times; increased co-transportation and ride-sharing, even in taxis; less private car ownership and more car pools, rental cars, taxis and freight forwarding agents; increased pedestrian and bicycle traffic; increased inter modality: bike or car and public transport; increased share of public transport on congested roads; improved opportunities to choose public transport which is not crowded; increased element of digitally mediated services at the expense of physical movements; increased accuracy and convenience in city traffic; smooth payment and information coordinated for all traffic in the city and thus an ubiquitously-available decision support for passengers and road-users .

\section{Sub-practices}

In order to illustrate the wide range of practices involved in shaping everyday travel we relied on SPT and the activity approach to identify five crucial aspects of travel in the ethnographic material. We here name these the social, material, improvisatory, combinatory respectively temporal aspect.

The social aspect points towards that, social relations and commitments actually 'produce' travel. The material aspect is also central, especially in social practice theory. It is through 
tools, objects and the built environment that practice is shaped and kept stable. Regarding the improvisatory aspect ethnographic research has shown that in large cities habitual car users, as well as public transport users, appreciate and justify their chosen travel modes in terms of flexibility and the possibilities of improvising activities, combinations of activities, itineraries, routes, stops etc. [17]. The combinatory aspect implies that, whether seen as activities or practices, the different doings of people in cities are to a high degree intertwined in a way that make most journeys the outcome of combined doings rather than single ones. Finally, the temporal aspect suggests that very different time horizons are normally at work when people plan and carry out their whereabouts, e $\mathrm{g}$ where to live or to manage much more immediate concerns such as how to rearrange travel plans for say an afternoon and evening of work and family life.

Addressing these aspects we believe the range of social practices related to urban travel could be in some sense pictured. To make our analysis more concrete we also identified a handful of specific practices at different 'endpoints' of that range. This we think could provide a basis for assessing and developing scenarios and measures for sustainable urban travel with ICT. Five sub-practices were identified in the ethnographic material as together covering the five aspects above in a way that we judged as being fruitful for the purpose of this paper. They can also be seen as important and sometimes problematic sub-practices of everyday travel that are of consequence for the spread of sustainable travel practices.

The five sub-practices are the following:

1) making vehicles prepared and available for a series of trips;

This sub-practice corresponds to the material aspect. Private vehicles need to be left or parked somewhere by the user after each trip. From the ethnographic studies [18, 19] it was evident that where and how to park the bicycle, scooter, car, pram etc. was an issue for travel planning in everyday life. In urban areas like the city of Stockholm where restrictions operate, free parking is scarse and congestion common most people mix different modes and do not bring the car, bicycle etc. 'everywhere'. Therefore it is necessary to plan the parking of vehicles to make them available for a subsequent trip, or even for a series of trips. This sub-practice highlights how the material aspects play an important and multifaceted role in travel related practice.

2) chauffeuring others (children, peers) and organize trips on their behalf;

This sub-practice corresponds to the social aspect. We chose it because it reflects that travel is primarily a social affair. City dwellers create activities, needs and trips together and for each other. In this way the sub-practice illustrates the social 'networking' behind that travel demand arises and persists [18]. That people plan and organize trips for others should thus be understood from a cultural point of view. To do things for others and exchange gifts, objects and services is a universal trait of societies. But it of course varies with time, place and group, what objects and services that are exchanged and expected from others. Travel is in any case something that is expected to be carried out and undertaken for the benefit of others and oneself. It is expected in relations such as parenthood, kinship, friendship and in more instrumental relations such as work life and belonging to the same club, school, parental group etc. This means that (planning of) a trip could be handed over to a counterpart in a culturally determined way.

3) managing trips that become disrupted;

This sub-practice corresponds to the improvisatory aspect. Trips can become disrupted for many reasons, e g internal when the traveler needs to change plans, or external, e g when a vehicle or a transport mode stops working in a specific area or connection. Urban dwellers are constantly learning and keeping up skills of using all kinds of resources to handle such disrupted trips [17].

\section{4) handling unique combinations of errands;}

This sub-practice corresponds to the combinatory aspect. To multi-task, and combine errands and commitments of different nature is related to complexity in everyday life [20]. This aspect has been elaborated in the 'activity approach' [4] and in Social Practice Theory [3].

5) making choices in life stage and career shifts;

This sub-practice corresponds to the temporal aspect. When people prepare and make changes in where they live, study, work etc. many of their travel-related practices could destabilize. People could in these shifts be open for practices they have not taken part in before etc. $[18,20]$. There is a long-term temporal aspect in that the new configuration of practice might be long-lasting after the destabilized phase. On the other hand the destabilized period in connection with the actual shift is relatively short term.

\section{Results and analysis}

The ethnographic studies showed how transport makes it possible for people to lead and enjoy urban life, but also how, at least in large cities, transport is a major area for self-service work and organizing as well as a frequent cause for worries and inconveniencies in daily life. Earlier research has also shown that urban travelers seem prone to respond to new technological possibilities, as well as to measures from transport providers and authorities [1]. This seems particularly true for opening new options for travel, accessibility and information exchange. How does the dependency, engagement and alertness in relation to transport, on the part of the citizens, relate to comprehensive transformation of urban transport systems in line with sustainable development? We found the above scenarios helpful in trying to answer such a question. For these ends we have regarded 'people carrying out practices' as a vital force in the system change dynamics, since the daily activities and doings in the city should be seen as what in practice upholds its systems [3]. Policy measures can, at least in some cases, be designed to affect changes in the practices, which in turn can change the system. This is at least the understanding and approach we advocate here. In line with this it is rather pointless to make minor adjustments in terms of addressing specific transport needs or even sub-practices of 
today, before finding out how practices and sub-practices may be important to change the overall dynamics. And to be able to address at least some aspects of overall change in this paper we rely on the selected scenarios. The scenarios we regard as representing the system, and sustainable development of it, that is our ultimate objective. On the basis of this we have chosen to regard practices decisive forces of change, in scenarios of sustainable development.

In line with SPT the doings (practices) should be seen as stabilizing but in flux, mostly imperceptibly but sometimes visibly, as with a new trend. Therefore we argue that it is in this change of practices that sustainability measures need to intervene in order to have lasting effects. We assume that such measures may in some cases succeed in changing practices, as part of reconfiguring the system.

We will in the following exemplify how the above subpractices could be included in the two selected scenarios.

Our first example concerns the sub-practice of making vehicles prepared and available for a series of trips. We argue that city dwellers might increasingly integrate 'smart-card' technology [15] in this sub-practice. Here we will specifically look at this sub-practice in relation to car pool vehicles. What does, and could, the sub-practice of making them prepared and available for a series of trips look like?

The booking systems for carpools currently operating in Stockholm are ICT-based. And the car pool members pick up as well as leave and park cars by using internet-connected smart card technology, installed in each car. Members find available cars, for any location and period of time, through using the booking system on the internet. As soon as the car is parked and logged out from with the smart card, another member can pick it up. Urry connected smart card technology with de-privatization of vehicles:

Cars more generally are being de-privatized through carsharing, car clubs and car-hire schemes. /---/ One important consequence is that if car users were not to own cars then car manufacturers would be responsible for shortterm car parking and for long-term disposal of 'dead' vehicles/---/. [15, p. 34]

By this last sentence Urry touches on the point in our paper of what makes it demanding for city dwellers today to keep vehicles available. Interestingly the material aspect of parking the car could be lifted off the car user, but another material aspect, reliance on the smart card technology is put onto the shoulders of the user. The smart card technology is mandatory in our example (car-sharing schemes operating today in Stockholm). There is no other way to pick up or leave the car, at least not without violating the rules of the car pool, or of the law. So what we see is how the practice of car-sharing already includes a variety of the sub-practice of keeping vehicles available for series of trips. In its car pool variety the subpractice is ICT-dependant, and the material aspect of this ICTdependency stabilizes the practice. There is no way around using the smart card technology, which makes members conform to the rules and routines. And the outcome is that vehicles are being kept available in a foreseeable way, for the common good of the pool members. This example also points at that in a future state of urban transport systems there could be found widespread ICT-based sub-practices that make the transport system function. But our point here is mainly on the transformative side. The sub-practice could be seen as an active force since it is a part of the transformation towards deprivatization of vehicles as well as towards increased use of 'smart' technology. What people do transforms the system of city travel. The specific variety of the sub-practice provides variation, in the sense of the word as a metaphor borrowed from ecology. In line with this metaphor restrictions and other conditions will provide selection. The practice of car-sharing ( with its bundled sub-practice of 'making vehicles available for series of trips') might eventually contribute to transformation of the system of travel.

Our second example of including sub-practice elements in scenarios concerns the sub-practice of organizing trips on others behalf. Here we didn't find any evidence of this subpractice being regarded in the handful of, otherwise suitable, scenarios we explored. To our minds it should be important to design scenarios and ideas of (ICT) services for trips that are produced through relations, rather than primarily for trips that come about through individual needs and decision-making. In the literature on ICT use in travel we see a clear bias towards so-called 'methodological individualism'. Technology, business and sustainability discourses in the field to a high degree focus on individual situations, decisions, facilities etc. at the expense of collective, social and relational ones.

Our third example finally concerns the sub-practice of making choices in life stage and career shifts. We argue that this sub-practice would be an important factor in achieving and sustaining an $I^{2} P S U T$-like system. Ethnographic studies have revealed that it is in those shifts that city dwellers actively find new arrangements for their everyday life and transport [18, 20]. This means that the sub-practice is one of those in which they would be most open to enter into a contract with transport providers. It would also include that through this sub-practice they could become co-producers of city transport by actively looking for agreements that enable this [14]. If this subpractice-based transformation should be written in the style of the $I^{2} P S U T$-paper [14] it could look like this:

City dwellers will especially in life stage shifts become more of co-producers of urban transport. In the subpractice of making such shifts they will look for opportunities to put their resources to use, in terms of skills, vehicles etc. Financial rewards will be important for many of them when entering into such co-production of transport. For others however, benefits in social terms or in terms of avoiding having to give up acquired habits and vehicles etc. would be equally important. Some urban dwellers would be more likely to join co-production in terms of joint transport and different groups of like-minded people (social communities). Others would expect, and catch, opportunities of becoming co-producers to private or public transport companies. Correspondingly, in the shift practice of moving house, forming family etc. city dwellers would also look for such contracts and agreements with transport 
providers, where they are not primarily co-producers but rather customers that sign up for reliable long term transport deals in terms of public transport, low risk of congestion for vehicles they drive themselves etc. In this way the sub-practice of making life stage shifts will be crucial for an I'PSUT-like system, in terms of that urbanites enter into the contracts and agreements that are important building blocks of such a system.

In relation to our analysis of other sub-practices we argue that once having signed up for a contract and committed themselves in terms of payment or work in joint transport, city dwellers will be very prone to learn and start to use the ICT functions of the system. This is similar to that car pool members have in practice shown that they accept and learn to master the ICT functionality that is necessary for using the shared vehicles. The ICT use thus becomes part of a package, and once the package is invested in, the user is likely to start using the ICT in the intended way in order not to 'waste her investment'.

With the two examples above we have shown how scenarios could be modified in line with our approach. We have only put two of the selected sub-practices to this use of modifying the scenarios. But in this limited format we hope this will be enough to understand our line of reasoning.

\section{Conclusion and discussion}

Use of ICT might contribute to transform travel in very different ways, since sub-practices for travel, in which ICT might be used, are of such diverse nature. We argue that, although aiming at selecting scenarios particularly adequate in terms of addressing ICT-based travel practice, the perspective of what people actually do and how this upholds and affects the transport system, was not sufficiently taken into account in the scenarios. This might reflect that this perspective is not sufficiently visible in policies and public debate concerning urban travel and ICT-use. In general policies and debate rather seem to focus on technology and institutional and political conditions. Consideration is seldom devoted to the notion that social practices could play a role in the dynamics between the different levels that technology, institutions and policies represent. To the extent that social practices are at all visible in the two scenarios, the perspective is too narrow, only certain types of practice could be discerned, not the entire span. However, this does not necessarily mean that the entire ideas behind the scenarios need to be questioned. Rather, the scenarios should to our minds be complemented by the role that practices among the urban dwellers play. This means that we see a need to do more research work on practice-based scenarios.

Could the development of ICT work as a key factor in the potential reshaping of the transport system? From the perspective of the methodology we used, we argue that if so this effect must to a significant extent come about through (sub-)practices, which change as the use of ICT in them becomes relevant, meaningful and attractive to use for urban dwellers. Remains the question of whether ICT use in travel is, or could become, ICT for sustainability. From the practice perspective our tentative answer is that material conditions (vehicles, ICTs etc.) and socially learned ways to handle these are fundamental to what possibilities and restrictions that actually emerge and operate. In terms of travel in a city there is always, and has to be, restrictions. We therefore argue that a remodeling of city traffic (such as in the two scenarios) is only likely to be in line with sustainable development when there are restrictions in terms of e.g. the city's infrastructure and physical form and/or restrictions in access to energy and other natural resources [18]. When limitations of environmental impacts are operating, new uses of ICT could contribute to ways of planning, using and providing city transport in line with sustainable development.

We claim that this should be taken into account by those who want to influence the development of urban traffic with ICT in a sustainable direction. On the basis of our analysis we propose the challenge for politicians, authorities and businesses to actively combat (development of) travel patterns and ICT use expected to lead to large environmental impacts. In the light of necessary restrictions opportunities should open for the above actors to support (sub-)practices involved in traveling and achieving accessibility, assessed to be related to relatively small environmental impact. Players on the arena of city traffic should to our minds actively support and create certain ICT uses, providing opportunities to travel and reach accessibility that is a) in line with ecologically sustainable development and b) in line with our conclusion that use could be expected to come about in terms of practices and sub-practices with transforming power.

Earlier research suggests that within an urban middle class the propensity is high to absorb easily accessible services to travel, plan and re-plan trips in advance as well as in real time [1]. There also seems to be some demand for new ways to pay. An important reason for that urban dwellers look for new ways to plan, travel, pay etc. seem to be commitments and objectives in their everyday lives. Accessibility and travel within the city plays a central role for most social practices. Since urban dwellers in many large cities experience that travel is not always satisfactory in the current situation [1] they show attention to new alternatives. Then it is consistent that there is interest and willingness to try new options. It is also logical to assume that there may be good acceptance and diffusion of new alternatives that prove to be able to solve the perceived need for mobility. Prevailing demand for new travel planning opportunities imply that factors such as indifference or lack of interest in relation to new transport services should not generally be seen as a barrier. We argue that as barriers should rather be seen 'mismatch', i.e. travel planning opportunities do not meet the needs of city dwellers, fits into their lives or reach them.

We propose that the authorities responsible for urban transport set goals not only for sustainability impacts but also for acknowledging, monitoring and trying to affect certain practices. Development of everyday social practice among city residents should be supported as an effective "force" in the development of the city's transport system [1], specifically including the development of ICT in relation to transportation. 


\section{Acknowledgment}

We gratefully acknowledge funding from Vinnova, Swedish Governmental Agency for Innovation Systems. In the project funding this research, we also work with the City of Stockholm, Ericsson, Samtrafiken, Stockholm County Council and Spacetime and the Swedish Transport Administration.

\section{References}

[1] M. Börjesson Rivera, G. Henriksson and M. Åkerlund (2012) Getting there and back again: Commuting and ICT in six cities across the globe. KTH Royal Institute of Technology, Series: TRITA-SUS, ISSN:1654-479X; $2012: 1$.

[2] A. Kramers (2014) Designing next generation multimodal traveler information systems to support sustainability-oriented decisions. Environmental Modelling \& Software. Issue 56, 2014. Pages 83-93.

[3] E. Shove and G. Walker (2010) Governing transitions in the sustainability of everyday life. Research Policy. Volume 39, Issue 4, May 2010, Pages 471-476.

[4] E. Shove and M. Pantzar (2005) Consumers, producers and practices: understanding the invention and reinvention of Nordic Walking. J. Consum. Culture, 5, 2005, pp. 43-64.

[5] M. Watson (2012) How theories of practice can inform transition to a decarbonised transport system. J. Transp. Geogr., 24, 2012, pp. 488-496.

[6] M. Fox (1995) Transport planning and the human activity approach. J. Transport Geogr. 1995, 3, 105-116.

[7] G. Henriksson, O. Hagman and H Andréasson (2011) Environmentally Reformed Travel Habits During the 2006 Congestion Charge Trial in Stockholm-A Qualitative Study. Int. J. Environ. Res. Public Health 2011, 8, 3202-3215.

[8] S. Maxwell (2001) Negotiating car use in everyday life. In: Car Cultures; Miller, D., Ed.; Berg: Oxford, UK, 2001.

[9] D. Miller (2001) Driven societies. In: Car Cultures; Miller, D., Ed.; Berg: Oxford, UK, 2001.
[10] M. Sheller (2004) Automotive emotions: Feeling the car. Theor. Cult. Soc. 2004, 21, 221-242.

[11] G. Sammer (2009) Non-negligible side effects of traffic demand management. In: Travel Demand Management and Road User Pricing: Success, Failure and Feasability; Saleh, W., Sammer, G., Eds.; Ashgate: Farnham, UK, 2009.

[12] I. Røpke and T. Haunstrup Christensen (2012) Energy impacts of ICT - Insights from an everyday life perspective. Telematics and Informatics, Volume 29, Issue 4, November 2012, Pages 348-361.

[13] A. Caragliu, C. Del Bo \& P. Nijkamp (2011) Smart Cities in Europe. Journal of Urban Technology Volume 18, Issue 2, 2011, pages 65-82.

[14] R. G. Hollands (2008) Will the real smart city please stand up? Intelligent, progressive or entrepreneurial? City: analysis of urban trends, culture, theory, policy, action. Volume 12, Issue 3, 2008, pages 303-320.

[15] J. Urry (2004) The 'System' of Automobility. Theory, Culture \& Society 2004 21: 25.

[16] A. Gullberg (2012) 'Så kan informationssamhällets landvinningar lösa stadstrafikens problem' (Using advances in the information society to solve urban-transport problems) PLAN 66 (5-6) pp. 64-71.

[17] H. Andréasson (2000) Resenärer $i$ bilsamhället: vardagligt resande $i$ kulturell belysning [dissertation]. Gothenburg: [Etnologiska institutionen, Univ.]; 2000.

[18] G. Henriksson (2008) Stockholmarnas resvanor - mellan trängselskatt och klimatdebatt (Travel Habits of Stockholmers congestion charging and climate debate; summary in English) [dissertation]. Lund: [Etnologiska institutionen, Lund Univ.]; Publ. by KTH Royal Institute of Technology, Series: TRITAINFRA-FMS $2008: 5$.

[19] Å. Nyblom (2014) Making plans or "just thinking about the trip"? Understanding people's travel planning in practice. Journal of Transport Geography 2014 35, 30-39.

[20] M. Godskesen (2002) Rutiner og brud i hverdagens transport. Et tekniksociologisk studie af børnefamiliers transport. [dissertation]. Copenhagen: [Danmarks tekniske Univ.]; 2002. 